\title{
ULTRASOUND DIAGNOSTICS OF THE SUPERCONDUCTING CABLE CONNECTIONS BETWEEN THE MAIN RING MAGNETS OF LHC
}

\author{
F. Caspers, T. Kroyer, J. Kulka ${ }^{1)}$, J.-P.Tock, L.R.Williams \\ CERN, Geneva, Switzerland
}

\begin{abstract}
As part of the LHC assembly program, the super-conducting magnets are interconnected after installation. Electrical continuity between the magnets is ensured via a specifically designed cable junction box which allows the cables to be electrically joined by an automated low temperature soldering technique. The electrical resistance and mechanical strength of the cable junctions depend on the quality of the soldered joint. An ultrasound diagnostic of the soldered junction has been developed to accompany the visual inspection and reinforce the quality control process. Non-standard ultrasound diagnostic techniques, without using matching liquids or gel in the harsh and congested working environment, applied to the sandwich structure of the cable junction box, which presents high ultra-sonic losses due to multiple scattering, have been developed. The equipment and methods implemented are described in detail, together with results of quality control tests made in the production environment.
\end{abstract}

${ }^{1)}$ AGH-UST, Cracow, Poland

CERN, 


\title{
ULTRASOUND DIAGNOSTICS OF THE SUPERCONDUCTING CABLE CONNECTIONS BETWEEN THE MAIN RING MAGNETS OF LHC
}

\author{
F. Caspers, T. Kroyer, J-P. Tock, L. R. Williams, CERN, Geneva, Switzerland \\ J. Kulka, AGH-UST, Cracow, Poland
}

\begin{abstract}
As part of the LHC assembly program, the superconducting magnets are interconnected after installation. Electrical continuity between the magnets is ensured via a specifically designed cable junction box which allows the cables to be electrically joined by an automated low temperature soldering technique. The electrical resistance and mechanical strength of the cable junctions depend on the quality of the soldered joint. An ultrasound diagnostic of the soldered junction has been developed to accompany the visual inspection and reinforce the quality control process. Non-standard ultrasound diagnostic techniques, without using matching liquids or gel in the harsh and congested working environment, applied to the sandwich structure of the cable junction box, which presents high ultra-sonic losses due to multiple scattering, have been developed. The equipment and methods implemented are described in detail, together with results of quality control tests made in the production environment.
\end{abstract}

\section{INTRODUCTION}

The next world-class facility for high energy physics, the Large Hadron Collider (LHC) [1] which will allow beams of protons and ions with a centre of mass energy of $14 \mathrm{TeV}$ to be brought into collision at nominal peak luminosity of $10^{34} \mathrm{~cm}^{-2} \mathrm{~s}^{-1}$, is being installed in a $26.7 \mathrm{~km}$ circumference tunnel at CERN. Among more than 7500 superconducting magnets comprising the LHC, 1232 main dipoles and 438 main quadrupoles have all been installed and are now being interconnected in the 8 arc regions occupying about $22.5 \mathrm{~km}$ of the machine circumference. Using state-of-the-art industrial techniques, each interconnect zone between 2 adjacent superconducting magnets is being completed. In the LHC arcs, more than 40'000 fusion welds on stainless steel piping and more than 60'000 junctions between superconducting cables are made to ensure the continuity of cryogenics, electrical and vacuum services from magnet to magnet and ultimately around the entire machine. All the main ring magnets in an LHC arc are electrically connected in series to a power source supplying currents up to $13 \mathrm{kA}$. The operational reliability of the complete machine depends directly on the diligence of the teams carrying out the interconnect assembly work according to specific procedures and heavily on the application of a strict quality assurance program to ensure that this quality is rigorously and continuously maintained.

\section{QUALITY ASSURANCE OF SOLDERED JUNCTIONS}

Each cable joint or splice is made inside a specifically designed cable junction box as shown in Figure 1 which allows the 2 cables together with 3 strips of tin/silver soldering alloy to be reliably and accurately superimposed and held firm while the soldering process is carried out.

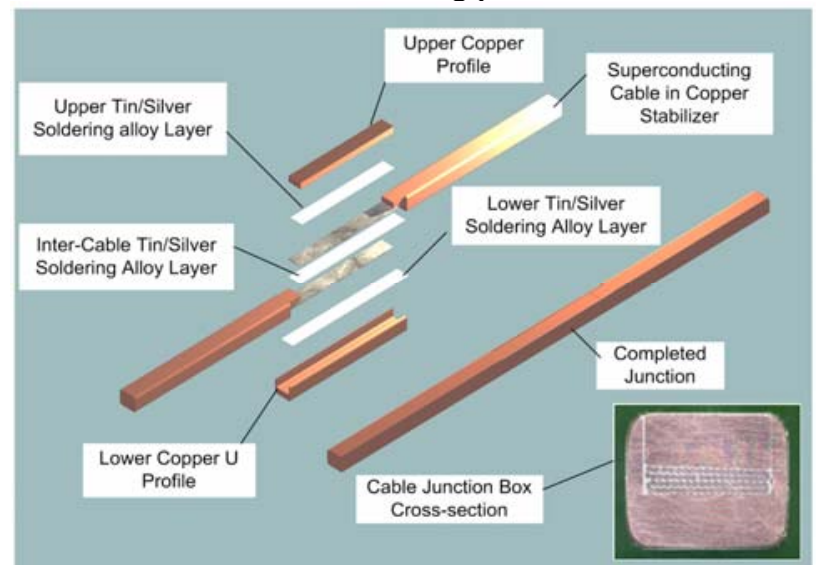

Figure 1: LHC Cable Junction Box

Whilst subjected to a controlled vertical compressive force ensuring intimate contact between all the component parts, the assembled junction is rapidly heated inductively to a fixed temperature where the added soldering alloy fuses with the residual layer binding the cable strands. The tight tolerances on the component parts of the box allow the molten soldering alloy to be retained inside by capillary action until the junction has cooled to below its solidification temperature. The heating power input, the compressive force and the temperature profile of the junction, are controlled and recorded over time and only if all post soldering data is normal is the junction accepted by the inductive soldering machine [3] as part of the standard Quality Control procedures. Key soldering parameters are interlocked and any excursion outside their normal envelope leads to automatic interruption of the soldering process and an alarm to be issued. Once the soldering process is complete the machine is removed and a detailed visual examination of the junction is made. Regular offline measurements on sample junctions are also made as part of the quality control plan. Junctions of excellent soldered quality systematically present an electrical resistance of the order of $0.15 \mathrm{n} \Omega$ at operating temperature. In comparison a sample junction where the flux and the additional soldering alloy strips have been voluntarily omitted before inductive soldering presents a resistance of 
about $0.3 \mathrm{n} \Omega$, a value still within the limit of $0.6 \mathrm{n} \Omega$ set for the LHC. The mechanical strength of a soldered junction may however be seriously undermined where it suffers from an absence or insufficiency of the alloy to be added during the soldering process. Although tight controls systematically regulate the soldering process, the absence, (omitted through operator error) or insufficiency, of tin/silver alloy in the junction is nowhere reliably detected. It is the possible omission of added alloy, leading to the undetected generation of mechanically weak soldered junctions that has motivated the use of ultrasound as a supplementary quality control method.

\section{PREVIOUS STUDIES}

Previous studies [2] on the in-situ non-destructive quality control of the LHC main magnet cable junctions using ultrasound have concluded that when probing the junction along a vertical axis this technique is inapplicable to the junction box environment which presents multiple interfaces of variable flatness between highly heterogeneous materials. In particular the multistrand multifilament structure of the cable leads to extensive multiple scattering and almost total dissipation of the ultrasound beam. Our first exploratory tests, when probing the junction along a vertical axis, both using one transceiver probe in transmission reflection mode and using 2 probes in transmission reception mode confirmed this.

\section{THE ULTRASOUND TESTING METHOD}

\section{Junction Quality}

The images in fig (2) show macrographic cross sections of (a) a cable junction box containing adequate alloy and (b) a junction box either severely deformed or containing inadequate alloy, where the vertical gap between the junction box components has not been completely filled by capillary action.

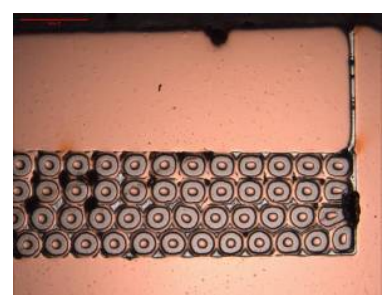

(a) good

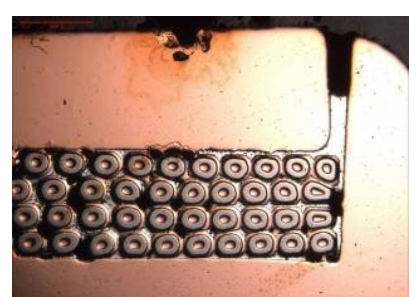

(b) bad
Figure 2: Macrographic Cross Section of a Cable Junction Box

An ultrasound diagnostic system probing along a horizontal axis above the cables and that is able to detect this vertical gap has been developed and implemented to reinforce the quality control process in the harsh and congested working environment of the LHC interconnect zone.

\section{The Ultrasound Diagnostic System}

Together with industry, micro-probes diameter $9.5 \mathrm{~mm}$ and length $9 \mathrm{~mm}$ with a beam diameter of $6 \mathrm{~mm}$ and a focal length of $10 \mathrm{~mm}$ have been developed. A commercially available carpenter's mini clamp has been modified and each jaw equipped with a micro-probe (Figure 3), one micro-probe is lodged directly into the jaw that passes between the bus bars and the other is assembled into a spring loaded enclosure that is then lodged in the other jaw. The contact face of the microprobes is equipped with a $0.6 \mathrm{~mm}$ thick Vulcolan ${ }^{\mathrm{TM}}$ high resilience polyurethane rubber membrane of hardness 50 Shore A that transmits the ultrasound energy and provides adequate surface coupling which only varies weakly with contact pressure on dry unclean surfaces, that in addition, may not be perfectly flat. The clamping device is applied perpendicular to the bus-bar and closed up to an end stop to ensure that all measurements are made under nearly constant spring-loaded clamping force. Figure 4 shows the rugged portable laptop based ultrasound diagnostic system connected to the clamping device applied to the main quadrupole bus-bars connected across an LHC interconnect zone

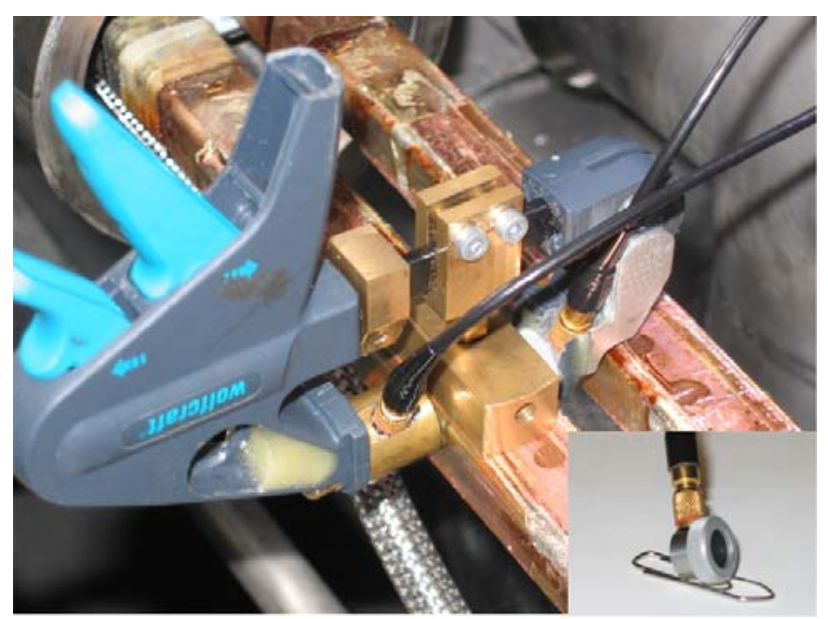

Figure 3: The clamp in position on an LHC Main Dipole Bus-bar and the Micro-Probe (inset)

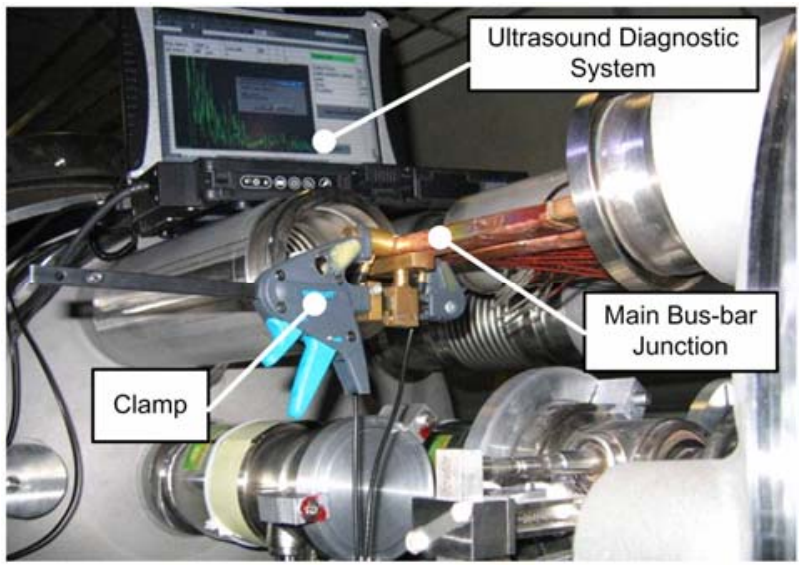

Figure 4: The Ultrasound Diagnostic System 


\section{The Measurement Method}

Two systems are in constant use making measurements in the LHC tunnel in the interconnect zones between magnets. The measurements must be tightly integrated into the production sequence and made immediately after a junction has been soldered so that, if rejected, immediate re-soldering followed by retesting may be carried out. Space for the probes and clamping system is severely limited by the presence of other previously interconnected equipment. Measurements are made at 4 equi-distant locations, along the length of the junction where the clamping system is positioned perpendicular to the bus-bar. The results of each measurement are automatically stored and a go no-go diagnosis of the junction quality at each location according to the pre-set limit is made. Measurements on all 6 bus-bars, 24 in total, take about 20 minutes to complete. A soldered junction is rejected if 3 out of the 4 measurements are below the acceptance limit.

\section{Data Output}

Figure 5 compares traces from two different junctions.

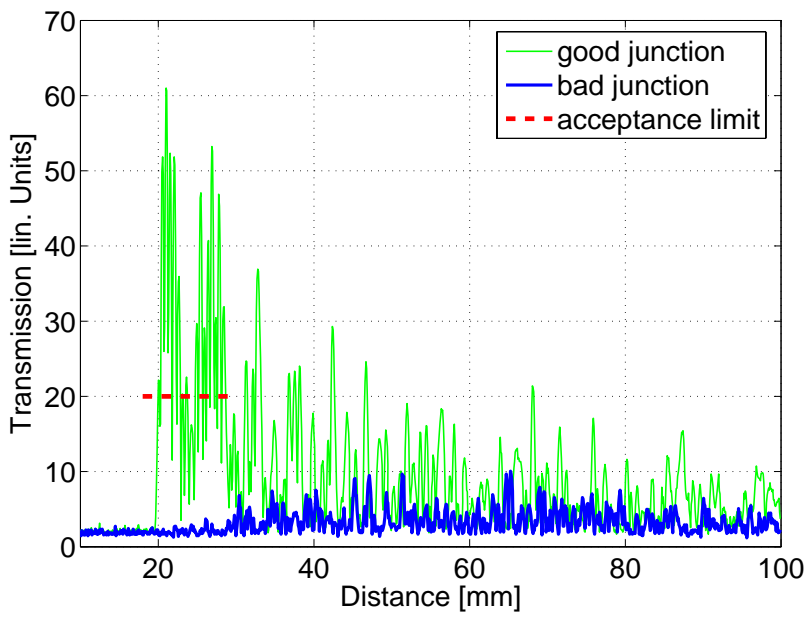

Figure 5: Comparison of Traces from a Good and a Bad Junction

The thin green trace is from a junction that was prepared under carefully controlled laboratory conditions and looks flawless visually. The typical features of such a "good" trace are (1) very good transmission at a distance corresponding to the junction thickness and (2) a few smaller peaks later on due to multiple reflections from the various interfaces in the set-up. The pre-amplification of the ultrasound device was set such that a good trace covers roughly the full display scale. The thick blue trace was taken on a junction that was soldered without any additional tin/silver alloy at all. The small amount of residual soldering alloy on the cable strands sticks the junction box together forming a "dry joint" whose mechanical strength is very low. Since no tin/silver alloy climbs up the sides of the junction, the transmission signal consists only of reflected waves that travelled around the lower $U$ shaped copper piece. The signal is much smaller and it arrives later than for a good junction. Based on these observations the trace acceptance limit was set as a combination of a time and amplitude window, allowing for small imperfections (dashed line).

\section{RESULTS}

From 2 operational measurement systems we have so far obtained a complete ultrasound diagnostic on soldered junctions on all 6 bus-bars in 195 interconnect zones. This represents a total of 1170 junctions measured and a total of 4680 individual measurements made. In three percent of the junctions measured, a maximum of two out of the four measurements made on each junction, were below the acceptance limit. These junctions are however acceptable. One junction with 3 measurements out of 4 below the acceptance limit has been found. According to LHC Quality Assurance Procedures this junction was declared non-conform and was immediately re-soldered with more tin/silver alloy being added to the junction, and re-measured. The repeat measurement showed the resoldered junction to be fully conforming. No junctions with 4 out of 4 measurements below the acceptance limit (corresponding to a case where additional tin/silver soldering alloy has been omitted) have been found.

\section{CONCLUSIONS}

A robust and non-contaminating ultrasound diagnostic system has been developed and implemented to measure the as-soldered quality of main bus-bar junctions in the interconnect zones of LHC. So far, a single soldered junction below the acceptance limit has been found and this corresponds to a failure rate of approximately $0.1 \%$.

\section{ACKNOWLEDGMENTS}

The authors would particularly like to thank the companies GE-Krautkramer (D) and Max C Meister (CH) for their advice on the implementation of the Ultrasound Diagnostic System and their help in the development and supply of the micro-probes. Thanks also go to T. Linnecar (CERN-AB) and L. Rossi (CERN-AT) for their support.

\section{REFERENCES}

[1] LHC Design Report Volume 1 "The LHC Main Ring”, CERN June 2004 ISBN 92-9083-224-0.

[2] A. Jacquemod, A. Poncet, F. Schauf, B. Skoczen, and J-P. Tock, "Inductive Soldering of the Junctions of the Main Superconducting Bus-bars of LHC" EUCAS2003 Sorrento2003.

[3] J.Ph. Tock, J.M. Balaguer, T. Colombet, A. Jacquemod, F. Laurent, C. Nambride, Y. Taffard, "Industrial tooling and methods for the junctions of the superconducting busbars in the interconnections between the LHC cryomagnets", EUCAS 2005, Vienna, 2005. 